\title{
Nova espécie de Hyla Laurenti do grupo de H. microcephala Cope (Amphibia, Anura, Hylidae) do nordeste do Brasil
}

\author{
Sergio Potsch Carvalho-e-Silva ${ }^{1}$, Ana M. P. Telles Carvalho-e-Silva ${ }^{2}$ \& Eugenio Izecksohn ${ }^{3}$
}

\author{
${ }^{1}$ Departamento de Zoologia, Instituto de Biologia, Universidade Federal do Rio de Janeiro. Caixa Postal 68044, 21944-970 \\ Rio de Janeiro, Rio de Janeiro, Brasil. E-mail: sergio@biologia.ufrj.br \\ ${ }^{2}$ Departamento de Ciências Naturais, ECB, Universidade Federal do Estado do Rio de Janeiro. 22270-000 Rio de Janeiro, Rio \\ de Janeiro, Brasil.E-mail: atelles@unirio.br \\ ${ }^{3}$ Instituto de Biologia, Universidade Federal Rural do Rio de Janeiro. Antiga Rodovia Rio-São Paulo, Km 47, 23851-970 Rio \\ de Janeiro, Rio de Janeiro, Brasil. E-mail: eizecksohn@netyet.com.br
}

\begin{abstract}
New species of the Hyla microcephala Cope group (Amphibia, Anura, Hylidae) from Northeastern Brazil. A new species, attributed to the group of Hyla microcephala Cope, 1886, is described. The new species is close to H. bipunctata Spix, 1824, and it is found in Quebrangulo, State of Alagoas, in Northeastern Brazil. The eggs and larvae are also described and some ecological information is added. The new species is compared with other species of the same group, and with species of closely related groups that occur in syntopy. With $H$. bipunctata, it shares the general form of the body, the yellow ventral coloration and the orange color of the thighs, but it is distinguished by the dorsal Paris-green coloration (beige in $H$. bipunctata), absence of a dorsal pattern, light red thighs, and ornamentation of the upper lip with two or three yellow stains instead of the characteristic aureolate of $H$. bipunctata. With $H$. elegans Wied-Neuwied, 1824, it also shares the general form of the body and the ventral and the thighs coloration, but it is distinguished by the smaller size, absence of a dorsal pattern and light red thighs. From H. oliveirai Bokermann, 1963, the new species is distinguished by the dorsal coloration, the ornamentation of the upper lip and larger size. From $H$. branneri Cochran, 1948, it differs by the presence of the stains in the upper lip (just one in $H$. branneri), absence of the dorsal pattern and green dorsal coloration, which is more intense. The tadpole of the new species is provided.
\end{abstract}

KEY WORDS. Alagoas, tadpole.

Na região nordeste do Brasil são conhecidas três espécies pequenas do gênero Hyla Laurenti, 1768 consideradas como pertencentes ao grupo de $H$. microcephala Cope, 1886: $H$. bipunctata Spix, 1824, H. branneri Cochran, 1948 e H. oliveirai Bokermann, 1963 (Cruz \& Dias 1991, Pugliese et. al. 2000). Outras pequenas espécies de Hyla também possuidoras de coxas imaculadas podem ser encontradas na região, como H. elegans Wied-Neuwied, 1824 e $H$. minuta Peters, 1872. No presente trabalho descreve-se uma espécie inédita, afim de $H$. bipunctata, obtida em Quebrangulo, Estado de Alagoas, Brasil.

\section{MATERIAL E MÉTODOS}

As cores referidas nas descrições são seguidas de seu código, entre parênteses, e estão de acordo com o catálogo de cores de SMithe (1975). As medidas são referidas em milíme- tros. Nas dimensões dos machos, fêmeas e girinos, são dadas as médias seguidas do erro padrão da média e, entre parênteses, da maior e da menor medida. Para medições dos girinos utilizou-se os estágios 31 a 33 da tabela de Gosner (1960) e a fórmula dentária está de acordo com Altig (1970). A fórmula da membrana interdigital segue a proposta de SAVAGE \& HeYer (1967) com modificação posterior de Myers \& Duellman (1982). Todos os 11 exemplares adultos, bem como o lote de larvas, foram obtidos em brejos com juncos, durante cinco campanhas, entre os meses de março de 1997 a novembro de 1999, em Quebrangulo, Alagoas, Brasil, e estão depositados na coleção científica do Departamento de Zoologia, Instituto de Biologia, Universidade Federal do Rio de Janeiro (ZUFRJ). As informações das desovas são de casais que desovaram em cativeiro. Para determinação dos girinos coletados, estes foram comparados com os girinos provenientes da desova obtida em cativeiro. 

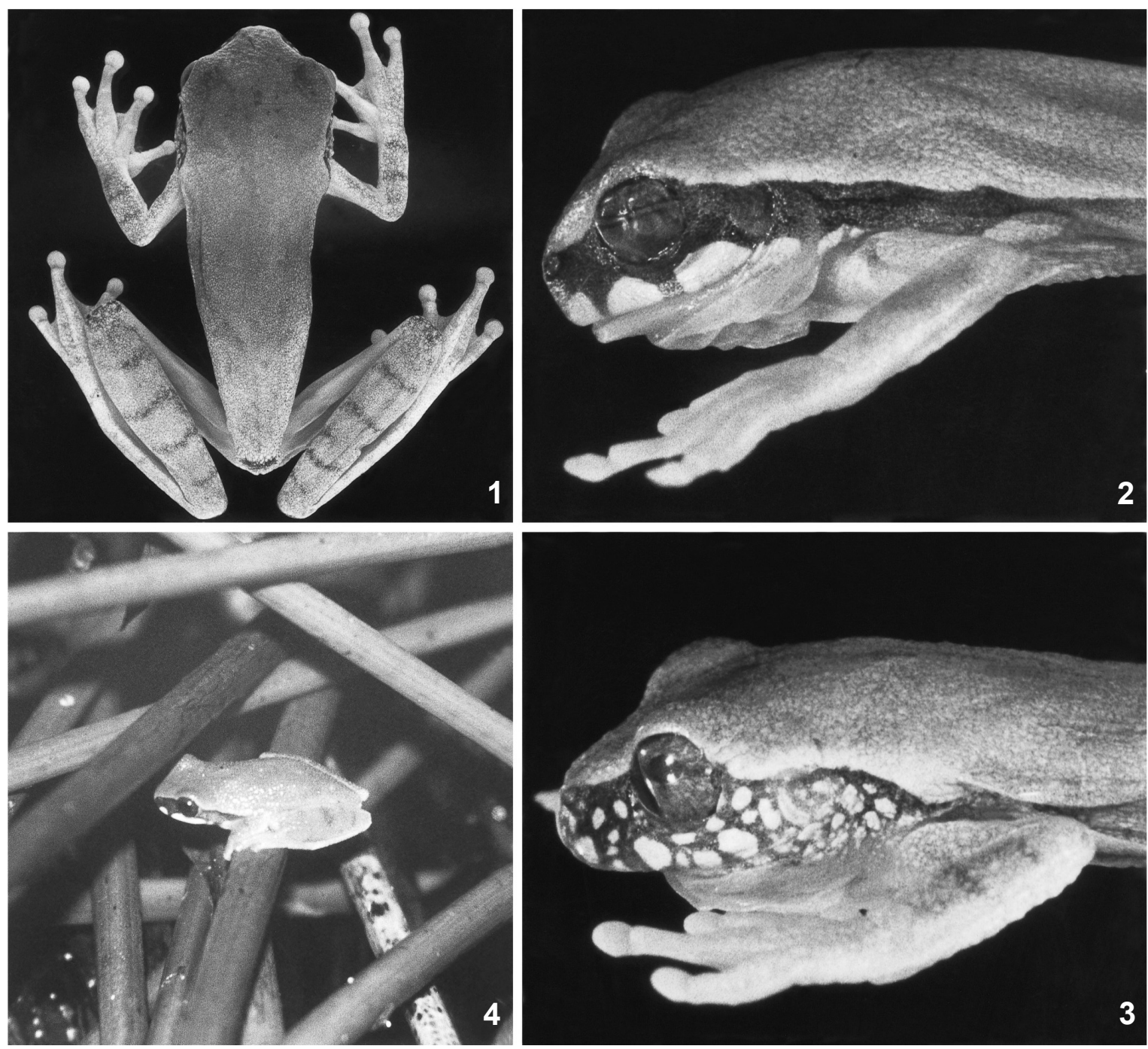

Figuras 1-4. (1-2) Hyla studerae sp. nov. holótipo macho, ZUFRJ 7180, de Quebrangulo, Alagoas: (1) em vista dorsal, (2) em vista lateral; (3) Hyla bipunctata em vista lateral; (4) Hyla studerae sp. nov. no ambiente, vocalizando no meio dos juncos, em Quebrangulo.

\section{Hyla studerae sp. nov.}

Figs 1,2 , 4 e $5-8$

Diagnose. Espécie pequena (CRA 20,8-24,8 mm nos machos e 26,8-29.6 mm nas fêmeas), atribuída ao grupo de Hyla microcephala, caracterizada por seu colorido dorsal verde (Paris Green, 63) durante o dia, abdômen amarelo e coxas alaranjadas, apresentando uma faixa lateral sépia (219), longitudinal, desde o focinho até o meio do flanco, com duas a quatro manchas amarelas de cada lado, no lábio superior e outras manchas menores (de duas a seis) do canto do lábio inferior até o meio do flanco.

Etimologia. Dedicamos a espécie à ornitóloga Anita Studer, que muito tem feito pela preservação das matas nordestinas.

Revista Brasileira de Zoologia 20 (3): 553-558, setembro 2003
Holótipo: ZUFRJ 7180, macho colecionado em Quebrangulo ( $\left.09^{\circ} 19^{\prime} 08^{\prime \prime} \mathrm{S}, 36^{\circ} 28^{\prime} 16^{\prime \prime} \mathrm{W}\right)$, no Estado de Alagoas, Brasil, março/1997, por Sergio P. Carvalho e Silva, Ana M.P.T. Carvalho e Silva e Aventino da Silva.

Parátipos coletados na localidade-tipo. Machos: ZUFRJ 7179 e 7181 (março/1997), por Sergio P. Carvalho e Silva, Ana M.P.T. Carvalho e Silva e Aventino da Silva; ZUFRJ 7286 a 7288 (novembro/1997), por Sergio P.Carvalho e Silva, Ana C.R. Alves e Aventino da Silva; ZUFRJ 7572 a 7583 e ZUFRJ 7585 a 7586 (junho/1998), por Sergio P.Carvalho e Silva, Ana M.P.T. Carvalho e Silva e Aventino da Silva; fêmeas: ZUFRJ 7284 - 7285 (novembro/1997) por Sergio P. Carvalho e Silva, Ana M.P.T. Carvalho e Silva e Aventino da Silva; ZUFRJ 7573 e 7578 (junho/1998), por Sergio P. Carvalho e Silva, Ana M.P.T. Carvalho e Silva e Aventino da Silva. 

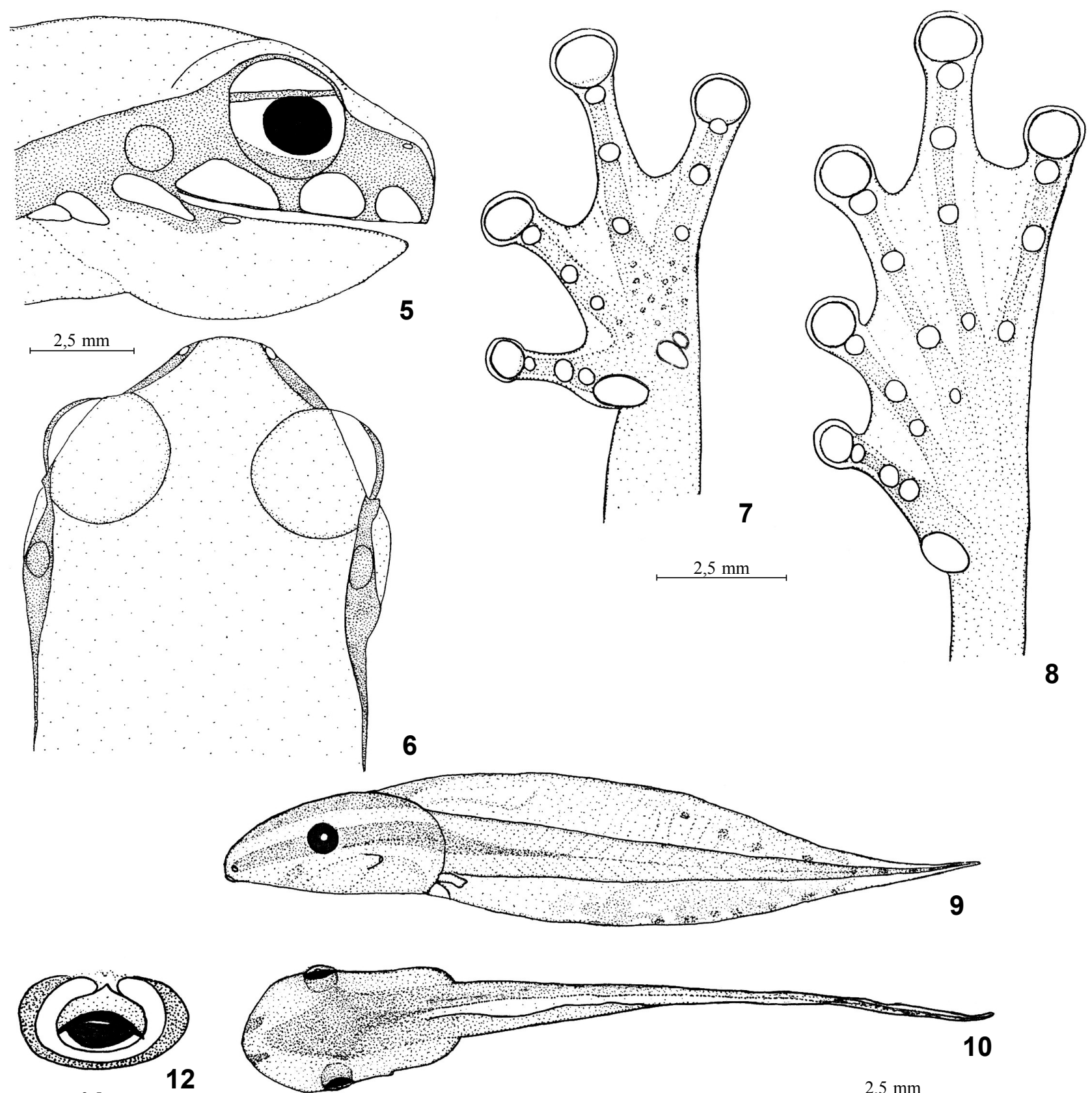

$\stackrel{0,5 \mathrm{~mm}}{\longmapsto}$

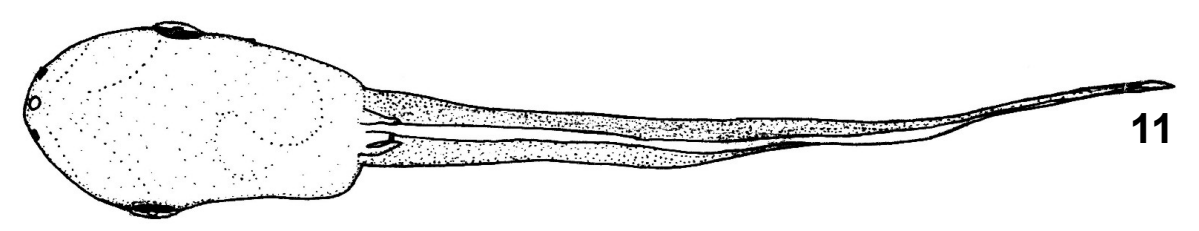

Figuras 5-12. Hyla studerae sp. nov. (5-8) Holótipo macho, ZUFRJ 7180: (5) cabeça em vista lateral, (6) cabeça em vista dorsal, (7) mão em vista palmar, (8) pé em vista plantar; (9-12) Girino, ZUFRJ 7627: (9) vista lateral, (10) vista dorsal, (11) vista ventral, (12) boca. 
Tabela I. Dimensões dos adultos de Hyla studerae sp. nov. de Quebrangulo, Alagoas.

\begin{tabular}{lccc}
\hline \multicolumn{1}{c}{ Dimensões } & Holótipo & Machos & Fêmeas \\
\hline Número de indivíduos & & 18 & 5 \\
Comprimento rostro-anal & 24,6 & $23,5 \pm 0,4(20,8-24,8)$ & $28,2 \pm 1,1(26,8-29,6)$ \\
Comprimento da cabeça & 8,0 & $7,9 \pm 0,2(7,3-8,7)$ & $9,0 \pm 0,6(8,0-9,7)$ \\
Largura da cabeça & 8,2 & $7,8 \pm 0,2(6,8-8,6)$ & $9,4 \pm 0,4(8,8-10,0)$ \\
Diâmetro do olho & 2,6 & $2,4 \pm 0,1(2,0-3,0)$ & $2,8 \pm 0,1(2,6-2,9)$ \\
Distância interocular & 4,8 & $4,8 \pm 0,2(4,2-5,7)$ & $5,4 \pm 0,2(5,1-5,6)$ \\
Distância interorbital & 3,1 & $2,5 \pm 0,1(2,0-3,1)$ & $2,9 \pm 0,6(2,0-3,5)$ \\
Distância do olho à extremidade do focinho & 3,1 & $3,0 \pm 0,1(2,6-3,5)$ & $3,4 \pm 0,5(2,4-3,9)$ \\
Distância do olho à narina & 1,8 & $1,7 \pm 0,1(1,3-2,4)$ & $1,9 \pm 0,1(1,7-2,1)$ \\
Distância internasal & 1,9 & $1,7 \pm 0,1(1,5-2,0)$ & $1,9 \pm 0,1(1,7-2,1)$ \\
Diâmetro do tímpano & 1,2 & $1,1 \pm 0,1(0,9-1,3)$ & $1,5 \pm 0,2(1,4-1,8)$ \\
Comprimento do úmero & 4,2 & $4,8 \pm 0,3(3,7-5,6)$ & $5,5 \pm 1,0(4,3-6,6)$ \\
Comprimento do antebraço & 4,0 & $3,9 \pm 0,1(3,5-4,3)$ & $4,6 \pm 0,2(4,3-4,9)$ \\
Comprimento da mão & 7,4 & $7,1 \pm 0,1(6,7-7,5)$ & $8,6 \pm 0,5(7,6-9,0)$ \\
Comprimento do fêmur & 12,7 & $12,5 \pm 0,2(11,1-13,2)$ & $15,1 \pm 0,6(14,2-15,9)$ \\
Comprimento da tíbia & 13,0 & $12,9 \pm 0,2(11,6-13,5)$ & $15,4 \pm 0,5(14,6-16,3)$ \\
Comprimento do tarso & 6,9 & $6,7 \pm 0,2(6,0-7,4)$ & $7,8 \pm 0,3(7,5-8,3)$ \\
Comprimento do pé & 11,7 & $11,0 \pm 0,2(10,0-11,7)$ & $13,5 \pm 0,7(12,3-14,5)$ \\
Diâmetro do disco do terceiro artelho & 1,3 & $1,0 \pm 0,1(0,7-1,3)$ & $1,3 \pm 0,1(1,1-1,4)$ \\
\hline
\end{tabular}

Material examinado. Hyla studerae: Brasil, Alagoas: Quebrangulo - adultos: ZUFRJ 7179-7181 26-29/3/97, 72847288 02/8/11/97, 7572-7586 17 a 21 /06/98; girinos: ZUFRJ 7627 17 a 21/06/98; desova: ZUFRJ 7289- (93 ovos). Hyla bipunctata - adultos: Bahia: ZUFRJ 3532, 5081, 5084; Espírito Santo: Linhares - ZUFRJ 212- 213, 905-910, 3183-3185, 4633. Hyla branneri - adultos: Espírito Santo: Linhares - ZUFRJ 2906, 3334; Bahia: Mucuri - 3516-3528, Itabuna - ZUFRJ 3744-3746. Hyla elegans - adultos: Bahia: Mucuri - ZUFRJ 3450-3455; Espírito Santo: Linhares - ZUFRJ 892-904. Hyla oliveirai - adultos: Bahia: Boa Nova - ZUFRJ 5257-5261.

Descrição do adulto (Tab. I, Figs 1, 2, 4 e 5-8). Cabeça tão comprida quanto larga; focinho levemente arredondado em vista dorsal, truncado em vista lateral; canto rostral distinto, arredondado; loros côncavos; narinas duas vezes mais afastadas do olho que da extremidade do focinho; distância olhonarina igual a $70 \%$ do diâmetro do olho; espaço interorbital maior que a largura da pálpebra superior; tímpano evidente, com diâmetro correspondendo a metade do diâmetro ocular; dentes vomerianos em duas séries entre as coanas; língua cordiforme, arredondada; saco vocal externo, único, subgular. Fendas vocais amplas, diagonais, laterais à língua. Dorso liso. Mãos com um calo carpal interno grande, ovalado; dois pequenos calos carpais externos; calos subarticulares arredondados desenvolvidos; calos acessórios pouco evidentes. Dedos em ordem crescente de tamanho, I < II < IV < III. Fórmula das membranas das mãos: I - II $(1-11 / 2)-(2-21 / 2)$ III $(2-21 /$ $2)-\left(2^{-}-2^{+}\right)$IV. Pés com um calo metatarsal interno ovalado, desenvolvido, calos metatarsais ausentes; calos subarticulares arredondados, calos acessórios pouco evidentes. Artelhos em ordem crescente de tamanho, I $<$ II $<$ III $<$ V $<$ IV. Fórmula das membranas dos pés: I $\left(1^{-}-1\right)-\left(1^{+}-2^{-}\right)$II $\left(1^{-}-1\right)-\left(1^{+}-2^{-}\right)$III (1$-2)-(1-2) \operatorname{IV}\left(2-2^{+}\right)-\left(1^{-}-1^{+}\right) \mathrm{V}$.

Coloração do adulto em vida. Toda a região dorsal verde (Paris Green, 63), incluindo cabeça, extremidade do focinho, tronco, tíbias, pés e antebraços, durante o dia e marrom (119 b) à noite. Uma faixa lateral sépia (219) com margem superior mais escura nos loros, passando pelo olho e se prolongando, afinando e esmaecendo, até pouco além do meio do flanco. Três a quatro manchas amarelas no lábio superior, podendo haver mais uma próximo à axila. Coxas alaranjadas (charmeorange, 16). Região ventral amarela, durante o dia, ficando mais esmaecida à noite. Íris castanha com pontuação acobreada.

Coloração do adulto no fixador. A coloração verde torna-se rósea, arroxeada ou azulada (entre as cores 76, 77 e 122), variando entre os indivíduos e esmaecendo com o tempo. A faixa lateral sépia torna-se mais esmaecida. As manchas labiais tornam-se brancas. Ventralmente os membros tornam-se amarelo ocre (yellow ocher, $123 \mathrm{c}$ ) e o tórax e abdomem tornam-se amarelo-pálido (chamois, $123 \mathrm{~d}$ ). Os olhos tornam-se opacos.

Descrição do girino (Tab. II, Figs 9-12). Girino de corpo alongado, focinho arredondado; cauda xificerca correspondendo a $73 \%$ do comprimento total, terminando em ponta afilada, sua altura correspondendo a cerca de 1,4 vezes a altura do corpo. Olhos laterais, distando entre si cerca de $90 \%$ da largura do corpo. Diâmetro dos olhos cerca de 16\% do compri- 
Tabela II. Dimensões dos girinos de Hyla studerae sp. nov. de Quebrangulo, Alagoas, $\mathrm{n}=4$, estágios 31 a 33 .

\begin{tabular}{lc}
\hline \multicolumn{1}{c}{ Dimensões } & Girino \\
\hline Comprimento total & $24,3 \pm 1,8(21,5-25,4)$ \\
Comprimento do corpo & $6,6 \pm 0,4(6,1-6,8)$ \\
Comprimento da cauda & $17,7 \pm 1,5(15,4-18,6)$ \\
Altura do corpo & $3,1 \pm 0,1(3,0-3,3)$ \\
Altura do cauda & $4,3 \pm 0,1(3,2-5,0)$ \\
Diâmetro do olho & $1,1 \pm 0,1(1,0-1,1)$ \\
Diâmetro da narina & $0,2 \pm 0,0(0,2-0,2)$ \\
Largura do corpo & $4,0 \pm 0,3(3,6-4,3)$ \\
Distância entre os olhos & $3,5 \pm 0,2(3,3-3,6)$ \\
Distância entre as narinas & $1,1 \pm 0,0(1,1-1,1)$ \\
Distância da narina à extremidade do & $1,1 \pm 0,1(0,9-1,2)$ \\
focinho & \\
Distância dos olhos à extremidade do & $0,2 \pm 0,1(0,1-0,2)$ \\
focinho & $4,7 \pm 0,4(4,3-5,2)$ \\
Distância do espiráculo à extremidade do & $0,6 \pm 0,1(0,5-0,6)$ \\
focinho & \\
Largura da boca &
\end{tabular}

mento do corpo, distando do focinho cerca de 39\% do mesmo comprimento. Narinas diminutas, arredondadas, localizadas na extremidade anterior do focinho, distantes entre si cerca de $28 \%$ da largura do corpo. Espiráculo sinistro, distante da extremidade do focinho cerca de $70 \%$ do comprimento do corpo. Tubo anal curto, voltado para o lado direito. Boca muito pequena, sub-terminal, sem dentículos ou papilas dérmicas, apresentando apenas o bico córneo. Maxila fina e mandíbula grossa, ambas arqueadas. Em torno do bico córneo há um sulco contornado por crista dérmica em forma de ferradura.

Coloração do girino em vida. Marrom-dourado em vista lateral, com uma faixa lateral marrom-escuro (Sepia 219), desde a ponta do focinho até o final do corpo, passando pelos olhos, até o final do primeiro terço da musculatura caudal. Abaixo dessa faixa, após o olho, o corpo é prateado opaco. Cauda marrom-claro levemente dourado, com a ponta transparente. Íris prateada, com anel claro em volta da pupila. Dorsalmente, corpo marrom-escuro, mais concentrado no centro. Ponta do focinho contornada por uma faixa arredondada marrom-dourado, até os olhos. Cauda marrom-dourado, porém mais clara. Ventralmente, corpo prateado com reflexos avermelhados na altura das brânquias e do coração até os olhos; após essa região até o final, prateado opaco. Nos estágios 30 e 31 , as patas posteriores, em formação, são bege-claro. Nos estágios 40 e 41 , as pernas se tornam marrons com faixas mais claras e o corpo apresenta-se marrom-claro com tons esverdeados. A cauda torna-se esverdeada e a íris começa a apresentar uma faixa longitudinal marrom acobreada, semelhante à do adulto.

Coloração do girino no fixador. Torna-se opaco, perdendo o brilho e os tons prateados e avermelhados.

Notas ecológicas. Durante os anos de 1997 a 1999, adul- tos foram observados em grandes concentrações, vocalizando sobre garmíneas e juncos, em poças em clareiras de mata, durante os meses de março e abril, logo após fortes chuvas. Cantavam expostos em noites escuras, ou entre a vegetação (Fig. 4) em noites de lua cheia. Vocalizaram durante todo o ano, mas a concentração de indivíduos era bem menor em períodos não chuvosos e nunca foram observados abaixo de $600 \mathrm{~m}$ de altitude. O amplexo é axilar e a desova, que obtivemos em cativeiro $(n=1)$, apresentou 415 ovos, tendo sido depositada na superfície da água. A eclosão se deu em cerca de dois dias. Os ovos variaram entre $1,1 \mathrm{~mm}$ e $1,2 \mathrm{~mm}$ e são envolvidos por duas membranas, variando de $2,2 \mathrm{~mm}$ a $2,4 \mathrm{~mm}$. Os girinos, tanto os provenientes da desova obtida em cativeiro, quanto os observados no ambiente, são muito ágeis, permanecem geralmente no meio da coluna d'água, indo à superfície ou ao fundo, movimentando apenas a ponta flageliforme da cauda que é xificerca.

Diferenciação. Hyla studerae sp. nov. parece muito afim a $H$. bipunctata, com a qual compartilha a forma geral do corpo, a coloração ventral amarela e as coxas de cor laranja, além das vozes semelhantes, mas se distingue prontamente pela coloração dorsal verde-Paris que se torna arroxeada, rósea ou azulada em conservador (bege em $H$. bipunctata), pela ausência do padrão dorsal, pelas coxas menos avermelhadas e pela ornamentação do lábio superior com duas ou três manchas amarelas em lugar do aureolado característico de $H$. bipunctata (Fig. 3). Com H. elegans, compartilha também a forma geral do corpo, a coloração ventral e das coxas, mas distingue-se pelo menor tamanho (CRA de H. elegans, segundo LuTz (1973): 20-27 $\mathrm{mm}$ machos e $29-36 \mathrm{~mm}$ fêmeas. CRA de $H$. studerae sp. nov.: 20,8-24,8machos e 26,8-29.6 mm fêmeas), pela ausência do padrão dorsal, pelas coxas menos avermelhadas e pela voz. De H. oliveirai, a nova espécie se separa pela coloração dorsal uniforme (H. oliveirai, segundo BOKERMANN (1963a), com faixa interocular e faixa longitudinal de cada lado do corpo brancas), pela ornamentação do lábio superior (ausente em $H$. oliveirai), pelo tamanho maior (CRA de $H$. oliveirai, segundo BOKERMANN (1963a): 16-17 mm machos e 19-20 mm fêmeas. CRA de $H$. studerae sp. nov.: 20,8-24,8 mm machos e 26,8-29,6 mm fêmeas) e pela voz. De $H$. branneri, difere pela presença das manchas no lábio superior (apenas uma em $H$. branneri), pela ausência de padrão dorsal característico de $H$. branneri e pela coloração dorsal verde mais intenso em vida. Difere ainda dessas quatro espécies pela coloração rósea a arroxeada no fixador. As cinco espécies ocorrem em sintopia na localidade-tipo de H. studerae sp. nov.

Os girinos, tanto os provenientes das desovas quanto os observados no local, apresentam as características das espécies do grupo de Hyla mircocephala, quais sejam, corpo alongado, flagelo terminal na cauda, ausência de dentículos córneos e de papilas na boca (Duellman \& Fouguette 1968, Cruz \& Dias 1991, Pugliese et al. 2000). O girino de $H$. studerae sp. nov. possui o olho proporcionalmente menor que o de H.bipunctata, cerca de $16 \%$ do comprimento do corpo $(19,7 \%$ em $H$. bipuncatata). Uma diferença marcante está na coloração em vida pois $H$. studerae sp. nov. possui o dorso do corpo marrom dourado com a cauda mais clara e o flagelo transparente enquanto $H$. bipuncatata possui o corpo marrom esverdeado com pontuações pretas, a cauda vermelho tijolo ornamentado de preto e o flagelo rosado com manchas marrom-escuro. A íris é prateada em ambas, porém a nova espécie possui um anel interno mais claro enquanto $H$. bipunctata possui manchas vermelhas com 
pontuações pretas na íris. Os girinos das duas espécies possuem uma faixa lateral marrom escuro desde a a ponta do focinho até a cauda, passando pelos olhos, e o ventre é prateado.

Discussão. O grupo de Hyla microcephala, definido por Duellman (1970), apresenta girinos de corpo alongado em vista lateral, olhos quase no meio do corpo, nadadeira dorsal começando no terço posterior do corpo, cauda marcadamente xificerca e disco oral desprovido de papilas, cristas ou fileiras de dentículos córneos (Pugliese et al. 2000). Como a larva de $H$. studerae sp. nov. apresenta essas características, nós a atribuímos a este grupo, no qual, de acordo com Cruz \& Dias (1991), mantemos $H$. bipunctata e $H$. meridiana.

Embora $H$. decipiens Lutz, 1925 e H. oliveirai tenham sido associadas ao grupo de H. microcephala (Bastos \& POMBAL 1996, POMBAL \& BASTOS 1998), suas larvas apresentam consideráveis diferenças que nos levam a considerá-las em um grupo separado, caracterizado por apresentar girinos ovóides em vista lateral; olhos no terço anterior do corpo; nadadeira dorsal começando no final do corpo; cauda com barras transversais escuras em fundo claro, com ponta afilada sem flagelo definido e disco oral com uma fileira de papilas (baseado em BokermanN 1963b, Pombal \& Bastos 1998, Pugliese et al. 2000). Este grupo (grupo de H.decipiens) é composto por $H$. decipiens, $H$. berthalutzae Bokermann, 1962, H. oliveirai e H. haddadi Bastos e Pombal, 1996.

\section{AGRADECIMENTOS}

Aos Srs Aventino da Silva e Marcus Senna pela ajuda nos trabalhos de campo; à Profa Ana Claudia Reis Alves pela criteriosa revisão do texto e pela ajuda nos trabalhos de campo; à Dra Anita Studer, da Fundação Nordesta, pelo financiamento das excursões. Somos gratos ao Conselho Nacional de Desenvolvimento Científico e Tecnológico (CNPq) pelo auxílio na compra de equipamentos.

\section{REFERÊNCIAS BIBLIOGRÁFICAS}

Altig, R. 1970. A key to the tadpoles of the continental United States and Canada. Herpetologica, Emporia, 26 (2): 180207.

Bastos, R.P. \& J.P. Pombal JR. 1996. A new species of Hyla (Anura: Hylidae) from eastern Brazil. Amphibia-Reptilia, Leiden,
17: 325-331.

Bokermann, W.C.A. 1963a. Nova espécie de Hyla da Bahia, Brasil (Amphibia, Salientia). Atas da Sociedade de Biologia do Rio de Janeiro 7 (2): 6-8.

. 1963b. Girinos de anfíbios brasileiros I (Amphibia, Salientia). Anais da Academia Brasileira de Ciencias, Rio de Janeiro, 35 (3): 465-474.

Cruz, C.A.G. \& A.G. Dias. 1991. Girinos do grupo "microcephala" do Estado do Rio de Janeiro (Amphibia, Anura, Hylidae). Revista Brasileira de Zoologia, Rio de Janeiro, 7 (4): 679-683.

Duellman, W.E. 1970. The Hylid Frogs of Middle America. Monographs of the Museum of Natural History, University of Kansas, vol. 1, 753p.

Duellman, W.E. \& M.J. Fouguette Jr. 1968. Middle American Frogs of the Hyla microcephala Group. University of Kansas Publications, Museum of Natural History, Lawrence, 17 (12): 517-557.

GosNer, K.L. 1960. A simplified table for staging anuran embryos and larvae with notes on identification. Herpetologica, Emporia, 16: 183-190.

LuTz, B. 1973. Brazilian Species of Hyla. Austin, University of Texas Press, XIX+265p.

Myers, C.W. \& W.E. Duellman. 1982. A new species of Hyla from Cerro Colorado, and other tree-frog records and geographical notes from Western Panama. Americam Museum Novitates, New York, 2752: 1-32.

Pombal JR., J.P. \& R.P. Bastos. 1998. Nova espécie de Hyla Laurenti, 1768 do centro-oeste brasileiro e a posição taxonômica Hyla microcephala werneri Cochran, 1952 e Hyla microcephala meridiana B. Lutz, 1952 (Anura, Hylidae). Boletim do Museu Nacional, N.S., Zoologia, Rio de Janeiro, 390: 1-14.

Pugliese, A.; A.C. Alves; \& S.P. Carvalho-e-Silva. 2000. The tadpoles of Hyla oliveirai and Hyla decipiens with notes on the Hyla microcephala group (Anura, Hylidae). Alytes, Paris, 18 (1-2): 73-80.

Savage, J.M. \& W.R. Heyer. 1967. Variation and distribution in tree frogs genus Phyllomedusa in Costa Rica, Central America. Beitrage Zur Neotropischen Fauna, Lisse, 5 (2): 111-131.

SmItHe, F.B. 1975. Naturalist's Color Guide. New York, American Museum of Natural History, 228p.

Recebido em 14.III.2003; aceito em 30.VIII.2003. 